\title{
BMJ Open A novel patient-reported outcome monitoring with clinical feedback system in bariatric surgery care: study protocol, design and plan for evaluation
}

\author{
Pål André Hegland (D) , ${ }^{1,2}$ Anny Aasprang, ${ }^{1}$ Ronette L Kolotkin, ${ }^{1,3,4}$ Christian Moltu, ${ }^{5}$ \\ Grethe S Tell, ${ }^{2}$ John Roger Andersen ${ }^{1,6}$
}

To cite: Hegland PA, Aasprang A, Kolotkin RL, et al. A novel patient-reported outcome monitoring with clinical feedback system in bariatric surgery care: study protocol, design and plan for evaluation. BMJ Open 2020;10:e037685. doi:10.1136/ bmjopen-2020-037685

- Prepublication history and additional material for this paper are available online. To view these files, please visit the journal online (http://dx.doi. org/10.1136/bmjopen-2020037685).

Received 13 February 2020 Revised 27 March 2020 Accepted 28 May 2020
Check for updates

(C) Author(s) (or their employer(s)) 2020. Re-use permitted under CC BY-NC. No commercial re-use. See rights and permissions. Published by BMJ.

For numbered affiliations see end of article.

Correspondence to MSC Pål André Hegland; palaheg@hvl.no

\section{ABSTRACT}

Background Consultations before and after bariatric surgery should include structured assessments of patients' health-related quality of life (HRQOL) and mental health. One way to conduct this assessment is to implement patient-reported outcome monitoring with a clinical feedback system (PRO/CFS).

Aim We will explore patients' and healthcare professionals' experiences when a PRO/CFS is an integrated part of bariatric surgery care.

Methods and analyses This is a design paper in which a PRO/CFS will be implemented in two bariatric outpatient clinics. All patients who have an appointment with a healthcare professional prior to, and 3 and 12 months after surgery, will be asked to complete six digital questionnaires measuring HRQOL, mental health, bowel symptoms and eating self-efficacy prior to each consultation. A digital summary report generated from the patient's responses will form the basis for the clinical consultation. A team of patient representatives, healthcare professionals and researchers will be involved in all phases of designing the PRO/CFS to ensure its relevance for clinical consultations. The patients' experiences will be explored with a generic 12-item questionnaire, developed for use in outpatient clinics, prior to and 12 months after bariatric surgery. We will conduct focus-group interviews with patients and healthcare professionals to explore their experiences when PRO/CFS is integrated into the consultations.

Ethics and dissemination Written informed consent will be obtained for all participants in the study. The project is approved by the Norwegian Centre for Research Data, Department of Data Protection Services (ref. no. 282738). The project has also undergone Data Protection Impact Assessments, both at Førde Hospital Trust and at St. Olav Hospital (registration no. 2016/3912). Data from the qualitative and quantitative studies will be kept in deidentified form in a secured research database, and the findings will be published in international peer-reviewed journals and presented at scientific conferences.

\section{BACKGROUND}

The term patient-reported outcome monitoring with a clinical feedback system (PRO/ CFS) refers to the systematic collection
Strengths and limitations of this study

- The involvement of patients and healthcare professionals in the design of the patient-reported outcome monitoring with a clinical feedback system (PRO/CFS) is a strength.

- A strength of the evaluation of the PRO/CFS is that it combines qualitative and quantitative methods to explore patients' experiences.

- A limitation may be differences in follow-up procedures in the two outpatient clinics.

- Another limitation is that the experiences of the healthcare professionals are explored using qualitative methods only.

of patient-reported outcome measures (PROMS) for immediate use in clinical consultations. ${ }^{1} \mathrm{PRO} / \mathrm{CFS}$ is synonymous with the term 'routine outcome monitoring and clinical feedback systems' (ROM/CFS), which is mostly used in mental health settings. For consistency in the present paper, we use the term PRO/CFS for both concepts/contexts. In PRO/CFSs, the patient responds to a set of questionnaires providing psychometrically valid information prior to consultation with a healthcare professional. ${ }^{1}$ The PROMS are collected before each clinical consultation, and clinical information from the patient, as well as comparisons to normative data, are fed back to the healthcare professional to be used in the clinical conversation. ${ }^{2} \mathrm{PRO} / \mathrm{CFSs}$ have been implemented in several healthcare services to assess mental health, somatic symptoms and health-related quality of life (HRQOL). ${ }^{3}$ A systematic review of qualitative studies found that healthcare professionals experienced clinical use of PROMS as useful when they were intended to guide patient management and when findings were presented clearly. Sufficient training in use and interpretation were also considered 
important. Barriers were lack of technical support, workload and when the PROMS were not considered relevant. ${ }^{4}$ In a recent qualitative study of patients' and healthcare professionals' experiences with PROMS, patients found that the PROMS helped them to address topics that were important for them. Furthermore, they could track their changes in symptoms and problems. Diseasespecific measures were considered to be most relevant. Barriers for both patients and healthcare professionals were lengthy questionnaires, and complicated summary reports that were difficult to interpret. This could serve as a hindrance for communication and professional relations. ${ }^{5}$ Common findings in both studies were the potential of PROMS to give patients a sense of control and facilitate patient-centred care.

PROMS can be collected on paper or digitally; however, a digital PRO/CFS has the advantage of providing instantaneous availability of the patient's responses, ${ }^{1}$ and, in novel systems, comparison to relevant norm data. An important feature for a PRO/CFS is the ability to combine nomothetic and idiographic approaches to understanding a given patient. In the nomothetic approach, individuals are characterised in terms of traits or dimensions that are based on mean scores of known groups. In the idiographic approach, the focus is on the individual and emphasises his/her unique personal experience, in $\mathrm{PRO} / \mathrm{CFS}$ concretised, for example, by measuring person-generated goals. ${ }^{6}$ The flexibility of PRO/CFS and its adaptation to the patient's needs and resources was found to be important in a qualitative study in mental health treatment, as patients experienced feedback as being part of treatment if their responses were discussed in the consultation. ${ }^{7}$ New approaches to PRO/CFS aim to contain both nomothetic and idiographic strategies through computer-adaptive testing.

Numerous studies from the field of psychotherapy have explored PRO/CSF both qualitatively and quantitatively, and therefore, they inform the use of PRO/CSF in bariatric surgery care. Meta-analyses and reviews show that $\mathrm{PRO} / \mathrm{CFS}$ have a small to moderate general positive effect on patient outcomes, particularly for patients not responding well to treatment interventions in its initial phases of psychotherapy. ${ }^{89}$ However, the results are not uniform, and some clinicians manage to use feedback better than others. ${ }^{10}$

To facilitate positive effects of $\mathrm{PRO} / \mathrm{CFS}$, the topics addressed have to be relevant for the group of patients. A review of systematic reviews found positive effects of bariatric surgery on HRQOL, especially with respect to obesity-specific and physical health concerns. ${ }^{11}$ Although improvements were seen in both the mental and physical domains of HRQOL, changes in the physical domains were greater.

Strategies to include assessments of psychological outcomes in the follow-up after treatment of obesity have been called for by patients ${ }^{12}$ and healthcare professionals. ${ }^{13}$ The European Association for the Study of Obesity guidelines for postbariatric surgery medical management recommend a structured mental health assessment, ${ }^{14}$ although they make no specific recommendations for how this assessment should be conducted. One strategy to structure the follow-up of patients after bariatric surgery is to implement a PRO/CFS to optimise HRQOL and mental health outcomes. ${ }^{3}$

In a recent overview of systematic reviews assessing the effectiveness of PRO/CFS on HRQOL, the authors found that the understanding and use of a PRO/CFS was quite variable across the included studies in both somatic and mental health services. The authors recommended that future studies should include detailed descriptions on how the concept of PRO/CFS is understood by the researchers $^{3}$ to clarify if the actual phenomenon of $\mathrm{PRO} / \mathrm{CFS}$ is being studied. Based on a systematic review of the PRO/CFS literature, the authors proposed three elements as being important for the successful implementation of PRO/CFS: (i) patient-reported outcomes should be routinely collected before all consultations, preferably digitally, (ii) the report should be available to both the patient and clinician during the consultation and (iii) patient-reported data and their implications should be discussed during the consultation. ${ }^{3}$ Considering these suggestions for the current study, we argue that in addition patient-reported outcomes should be understood in the context of normative data-that is, how similar or different is this patient's scores from other patients with the same condition and/or the healthy population.

Integrating knowledge from the mental health field with experiences from somatic health services, this project aims to develop, implement and evaluate a PRO/ CFS for use in an outpatient setting in bariatric surgery in Western Norway. The purpose of this article is, therefore, to describe the development and implementation of the $\mathrm{PRO} / \mathrm{CFS}$, and our plan to evaluate the feasibility of the $\mathrm{PRO} / \mathrm{CFS}$ in bariatric surgery care. We hypothesise that using a digital PRO/CFS will add value to the collaborative relationship between patients and healthcare professionals in bariatric surgery care. The following research questions will be addressed in the planned study:

1. How do patients experience the clinical consultation when a PRO/CFS is an integrated part it?

2. How do healthcare professionals experience the clinical consultation when a PRO/CFS is an integrated part of it?

\section{METHODS}

\section{Design}

This paper describes the design of a PRO/CFS that will be implemented in the bariatric surgery outpatient clinics at Førde Hospital Trust and St. Olav Hospital Trust in Norway, and a plan for evaluating the feasibility of this intervention. The aims of the planned study are to evaluate experiences of patients and healthcare professionals with the consultations, using quantitative and qualitative methods. The quantitative assessment of patients' experiences started August 2019 and will be completed during 
Table 1 The organisation of the bariatric surgery follow-up in the two outpatient clinics

\section{Helse Førde Hospital Trust}

First contact with the specialist healthcare services

Patients attend a one-day preoperative information course. A bariatric surgeon, nutritionist, physical therapist, psychologist and bariatric outpatient nurse provides information about BS and a lifestyle intervention programme during the course.

Patients who seeks BS as preferred treatment are referred to a Patients eligible for BS attend mandatory group sessions psychologist for a mental health assessment before surgery.

The patients meet for a preoperative consultation with a bariatric surgeon and a nurse, where the best choice of treatment for the patient is decided.

\section{Follow-up after surgery}

At:

- 6 weeks, telephone consultation with a nurse;

- 3 months, consultation with a bariatric surgeon and a nurse;

- 6 and 12 months, consultation with a nurse;

-12-18 months, a one-day group-based course at the outpatient clinic;

- 24, 60 and 120 months, consultation with a nurse. Other healthcare specialities are consulted as needed.

The GP is intended to follow up the patient yearly in between.

\section{St. Olav Hospital Trust}

First contact with the specialist healthcare services

Patients seeking BS have a consultation with a physician and a nurse during which the choice of treatment is decided.

over 4 months, where a physician, psychologist, nurse and nutritionist educate the patients.

After the mandatory group sessions, the patient will meet with the surgeon for preoperative information.

\section{Follow-up after surgery}

At:

- 1 week, telephone consultation with a nurse;

- 6 weeks, consultation with a bariatric surgeon;

- 1-3 months, one group session with a nutritionist;

- 6 months, consultation with a nutritionist;

- 12 months, consultation with a medical doctor;

- 24 months, consultation with a medical doctor;

- 36, 48 and 60 months, consultations with a nurse.

$\mathrm{BS}$, bariatric surgery; GP, general practitioner.

December 2022. For the qualitative inquiries, we plan to recruit informants and conduct the focus-group interviews during autumn 2020.

\section{Patient and public involvement}

Two patients have been involved in choosing the appropriate questionnaires in the $\mathrm{PRO} / \mathrm{CFS}$ and have also been involved in the pilot-testing of the PRO/CFS.

\section{The standard bariatric care programme at the outpatient clinics}

Patients with severe obesity are referred from their general practitioner (GP) to the bariatric outpatient clinic. An evaluation of the best choice of treatment to the individual patient (surgical vs non-surgical treatment) is then performed by the specialist healthcare services. Furthermore, the preparation before surgery and the follow-up consultations in the two outpatient clinics are somewhat different from each other (see table 1 for further details).

\section{Designing the PRO/CFS}

Because user involvement is a key element in evidencebased medicine,${ }^{15}$ to ensure that the research is relevant and ecologically valid for users of the services, ${ }^{1617}$ we had a panel consisting of two patients who had undergone bariatric surgery, and one nurse working at the bariatric outpatient clinic to assist in the design of the PRO/CFS. The panel also included three researchers with experience in obesity treatment and research. One of the nurse researchers $(\mathrm{PhD})$ had a combined position in obesity outpatient clinic and research, whereas the other two (PhD student and Professor) worked as researchers.

The purpose of the included questionnaires was that they should be clinically relevant for both patients and healthcare professionals, facilitate patient-centred care and improve patient-clinician communication. To achieve this end patients, outpatient nurse and researchers held a workshop where the relevance and utility of each of the questionnaires were assessed. Special attention was paid to whether the questionnaires included aspects that the patients considered important for the follow-up, or if any questionnaires were found offensive for the patients.

\section{Intervention}

The theoretical basis for the intervention in this study is Feedback Intervention Theory (FIT) ${ }^{18}$ which considers it important to have a discussion between the patient and healthcare professional, and focuses on the gap between the patient's current situation and an established benchmark. FIT is a theory commonly applied to the field of PRO/CFS implementation. ${ }^{2}$

The intervention is a digital $\mathrm{PRO} / \mathrm{CFS}$, composed of questionnaires assessing HRQOL, ${ }^{19-21}$ mental health, ${ }^{22}$ bowel-specific symptoms ${ }^{23}$ and eating self-efficacy ${ }^{24}$ (see online supplementary file 1 for details on the questionnaires).

The invitation letter asks the patients to complete the $\mathrm{PRO} / \mathrm{CFS}$ prior to the consultation with a healthcare professional. The patients can enter the PRO/CFS via a link in the hospitals' web page, on either their own 
computer or a tablet in the waiting area in the clinic. To access the system, we will use the highest public data security-level in Norway-password-protected two-factor authentication system. If the patient does not have access to this system, the healthcare professional can generate a unique login for the patient. The PRO/CFS platform is delivered by the manufacturer CheckWare in Norway.

\section{Method for completing the PROMs}

Using the PRO/CFS-software, the patients answer a set of questionnaires. As not all questionnaires include the possibility for the patient to answer Not relevant/Do not want to answer, the patient can choose not to respond to an item in all questionnaires except the Norse Feedback. If the patient has reported one or more Not relevant/Do not want to answer in the Norse Feedback, the average of the other items in the scale is shown in the summary report. In the other questionnaires, the average of the items is shown if $>50 \%$ of the items are completed. On completion, a digital report becomes available for the healthcare professional. The report represents a summary of each questionnaire, colour-coded red, yellow and green (see online supplementary file 2 ). The responses in the red areas are domains in which the patient has the most concerns. The yellow responses are domains where the patient has some concerns and green responses are domains where the patient has few or no concerns. The thresholds for the colour categories are defined by normpopulation standards, where available, or by the clinical judgement of the research group (see online supplementary file 1 for further details). The thresholds are set to be sensitive for impairment, as we find it important that the healthcare professionals do not overlook any concerns the patient may have. Prior to the consultation, the healthcare professional will be provided with an overview of the patient's responses, and during the consultation, the patient and the healthcare professional are instructed to begin by discussing the domains in which the patient has reported the most concerns (see figure 1 ). The different questionnaires incorporated into the $\mathrm{PRO} / \mathrm{CFS}$ will be evaluated separately, within their separate interpretative frameworks during the consultations. The patient's responses from the current and earlier consultations are visualised in the same report, to enable evaluation of the patient's progression in treatment. The patient and the healthcare professional will agree on how to deal with any concerns that cannot be addressed adequately during the initial consultation. In most cases, the solution will be a detailed clinical report to the GP. If the concerns are urgent, the GP will be called to discuss further. In other cases, the healthcare professional may refer the patient to consult with the specialist healthcare services if this level of expertise is required. Another alternative is to offer the patient a new consultation with the healthcare professional at the obesity clinic.

\section{Implementation phase}

We will start the implementation of the PRO/CFS at Førde Hospital Trust.

Focus on change management is vital for a successful implementation of this intervention. To that end, we have secured management commitment in both outpatient clinics. Furthermore, informational sessions, training and follow-up is planned for all healthcare professionals at project launch and throughout the programme. We have planned up to 3 days of training of the healthcare professionals, depending on the needs of the individual. Average training duration will be measured.

Prior to implementing the project, a team of patients, outpatient nurses, and researchers will fill out the PRO/ CFS to test the functionality and feasibility of the system. The outpatient nurse will receive further training to interpret the results from the report, and learn how to incorporate these findings in clinical conversations with the patient. Such training is recommended for a successful implementation. ${ }^{25} 26$ Two researchers serving as proxy patients will conduct the training of the nurse. This training will have special attention on interpreting the mental health findings, as the assessment of the patient's mental health is more thorough than the healthcare

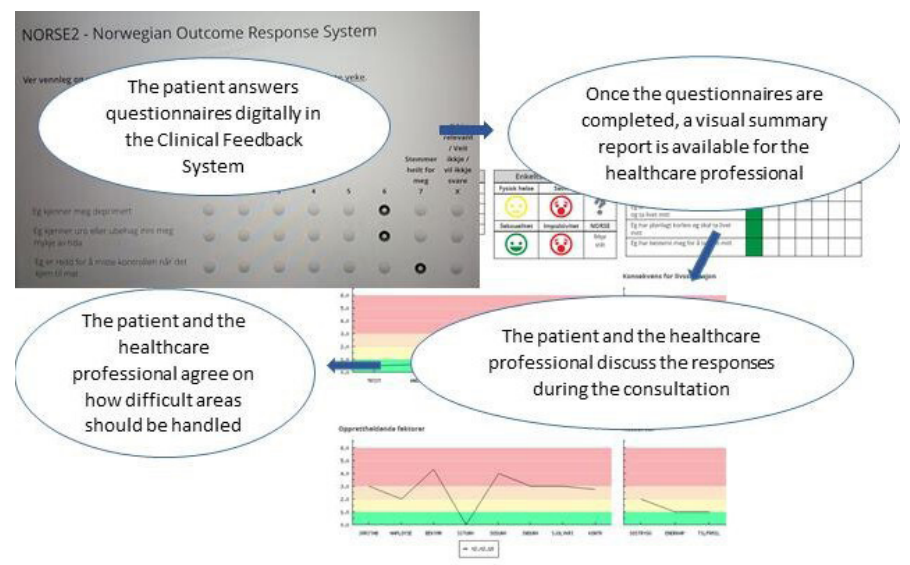

Figure 1 Flow diagram of the process and use of the patient-reported outcome monitoring with a clinical feedback system (PRO/CFS) in consultation between the patient and the healthcare professional in the bariatric outpatient clinic at two Norwegian hospitals. 
professional has experience with. After implementation starts, the first author will join the initial patient consultations to guide the outpatient nurse in the interpretation and use of the PRO/CFS. Moreover, a person hired by the hospital to work on the implementation of PRO/CFS will be available to answer technical questions. The first author also will continue to join selected consultations to: evaluate adherence to the incorporation of patientreported information in the clinical consultations; support the healthcare professional; serve as a fidelity check of how the feedback is being incorporated into the consultation. Such follow-up over time has been shown to be important to facilitate successful implementation in complex systems. ${ }^{27}$ At St. Olav Hospital Trust, the implementation of the PRO/CFS will also include training of all involved healthcare professionals in how to interpret the report, and to incorporate these findings in the conversation with the patient. The healthcare professionals will have the assistance of a researcher with experience with the PRO/CFS, as well as technical support. Fidelity to the use of PRO/CFS is defined as the healthcare professional and the patient studying the summary report together and discussing the patient's responses during the consultation. Adherence to fidelity will be evaluated by the researcher attending the consultations. Furthermore, the healthcare professionals will complete a fidelity checklist for a random selection of consultations, and a separate question will be added to the Generic Short Patient Experiences Questionnaire (GS-PEQ) asking the patient whether feedback was given during the consultation.

\section{Outcomes and analyses in the feasibility study}

The aim of the study is to assess the feasibility of this $\mathrm{PRO} / \mathrm{CFS}$ in bariatric surgery care. We plan to accomplish this by assessing patients' experiences using both quantitative and qualitative methods, and the healthcare professionals' experiences using qualitative methods. We will collect data on number of planned consultations, attrition to the follow-up, whether the patient has completed the PRO/CFS prior to the consultation and the presence of any missing data within the completed $\mathrm{PRO} / \mathrm{CFS}$. In addition, we record the proportion of consultations requiring contact with, or referral to, other healthcare specialties to assess how the PRO/CFS affects the course of the follow-up. The qualitative interviews and the quantitative findings of the experiences will be used to evaluate the utility of the PRO/CFS. Indications that $\mathrm{PRO} / \mathrm{CFS}$ is operating as intended are if satisfaction with consultations are high as measured with GS-PEQ, and if the overall impression from the qualitative interviews is positive. An overall impression of dissatisfaction in the qualitative interviews or low scores in the GS-PEQ indicates a need for refinement of the PRO/CFS.

Quantitative assessments and analyses of patient experiences The GS-PEQ ${ }^{28}$ is a set of 10 core items developed to measure patient-reported experience measures in different somatic and psychiatric health services in
Norway. We will use the version validated for use in somatic outpatient services, which consists of 12 items measuring experiences regarding information and communication (eg, Did the clinicians talk to you in a way that was easy to understand?), whether the patient had any influence on the treatment (eg, Were you involved in decisions regarding your treatment?) and the perceived benefit from the treatment (Overall, was the help and treatment you received at the institution satisfactory?). The items are rated on a 5-point scale ranging from Not at all to To a very large extent. The items will be analysed separately, without a total score, and the responses To a large extent and To a very large extent will be regarded as an indication that the PRO/CFS is operating as intended. We will pay special attention to the following four questions: Did the clinicians talk to you in a way that was easy to understand?; Did you perceive the treatment as adapted to your situation?; Were you involved in decisions regarding your treatment? and Overall, was the help and treatment you received at the institution satisfactory? as indicators of the utility of the PRO/CFS. This questionnaire will not be incorporated in the PRO/CFS but will be answered by pen and paper after the consultation and delivered to the healthcare professional in a closed envelope.

The patients' experiences will be presented through descriptive statistics. Further, their associations in relation to gender, age, body mass index, HRQOL, complications of the surgery and bowel symptoms (from the Gastrointestinal Symptoms Rating Scale) will be investigated using univariate and multivariable regression analyses. Analyses will be conducted on patients with at least 1 year of follow-up after surgery. Given sample size $=100$, power $=0.8$ and $\mathrm{p}=0.05$, the study would be able to detect an unadjusted standardised coefficient $=0.27$ ( $\mathrm{G}^{*}$ power 3.1.9.4). This corresponds to a small effect size. ${ }^{29}$ The computer software IBM SPSS statistics ${ }^{30}$ will be used for the statistical analyses.

\section{Qualitative inquiries into patients' and healthcare professionals' experiences with PRO/CFS}

We plan to conduct separate focus group interviews of patients and healthcare professionals in the obesity outpatient clinic to explore their experiences when the $\mathrm{PRO} / \mathrm{CFS}$ is an integrated part of the consultation. Focus group methodology is particularly apt for health service research, as different participants can expand on each other's perspective in formulating their experiences in the shared context. The participants for the focus group interviews will be recruited from obesity outpatient clinics at the two hospitals. The patients will be recruited by clinical staff, whereas the healthcare professionals will be recruited on an information meeting, followed by an invitation on email. We plan to report how many patients and healthcare professionals are invited to the study, and how many accept/decline to participate.

We plan four focus group interviews with patients, two from each outpatient clinic, and one focus group with healthcare professionals in the initial data collection. We plan to accrue six to eight participants in each group. 
Follow-up focus groups will be implemented after 12 months. The interviews will be audio-recorded and transcribed verbatim for analyses.

A researcher will serve as the moderator of the focus groups, and a co-researcher will be present to take notes and handle the audio-recording. The role of the moderator is to make sure that all participants get to tell their story, to stimulate interaction between different perspectives in the group and to make sure that the conversations relate experiences relevant to the research questions. The interviews will be implemented within the hospital areas, but not in the outpatient clinic. This will be done to allow the participants to convey their own perspectives. We will prepare a brief introduction stating the aim of the conversation, and a schedule of open-ended questions for the group to discuss. An interview guide will be developed in collaboration with patient-representatives and an outpatient nurse (see online supplementary file 3 for interview guide). We will conduct a pilot interview to evaluate the relevance of the interview guide.

For analysis of the transcribed data, we plan to apply systematic text condensation (STC), ${ }^{31}$ where we will synthesise the data in four steps. STC is a structured qualitative method for analyses that is well documented for research in medical settings. The first step is to get an overall impression of the transcribed text to get familiar with the content and create themes. The next step is to identify units of meaning and code these to sort out units of text related to each other. The third step is to abstract the units of text into a condensate of units from the different participants. In the final step, the content from the condensates are synthesised, which means that the condensates are interpreted by the researches. The interpretation must be loyal to the voices of the participants, and at the same time be influenced by the researcher's interpretation. ${ }^{31}$ We will have two researchers conducting the analyses separately and come to agreement through discussion. In addition, we will present the anonymous transcribed interviews and the preliminary analyses to an experienced qualitative researcher, to perform the role of independent critical auditor, to secure correspondence between results and data. ${ }^{32}$

\section{DISCUSSION}

The purpose of this project is to implement a $\mathrm{PRO} / \mathrm{CFS}$ in bariatric surgery care. We aim to explore the patients' and healthcare professionals' experiences with consultations, where PRO/CFS is an integrated part of the clinical conversation.

\section{Structured assessment}

This project is a novel approach to meeting the challenges of a structured assessment of patients' HRQOL and mental health, as called for by both patients and healthcare professionals, and highlighted in guidelines for follow-up after bariatric surgery. ${ }^{12} 14$ The measures of mental health symptoms are more thorough than the measures previously used in this patient population in Norway. The multidimensionality of Norse Feedback ensures that clinical constructs are not evaluated alone but are viewed through their relationships with other clinical dimensions within the measure, the patient's ability to change and social support. This means, for example, that two patients who have the same symptom load on depressive symptoms but experience their situations differently because one has good social support whereas the other has not, are viewed differently. Furthermore, for a patient with an elevated suicidality score, the situation will often be more concerning if he or she at the same time has elevated scores in the scales Hopelessness and Social avoidance, than if he or she scores low on these same scales. We hypothesise that this will allow for a more detailed understanding of mental health processes and better decisions in the clinical conversation.

Because the PRO/CFS incorporates questionnaires assessing diverse issues related to bariatric surgery, such as bowel symptoms and eating self-efficacy, the battery of questionnaires will make the PRO/CFS clinically relevant to the patients and healthcare professionals.

\section{PRO/CFS methodology}

To simplify the integration of PROMS in clinical consultations, we have chosen a digital PRO/CFS, as this has the advantage of instant and easy to interpret summary reports. ${ }^{1}$ The summary report from the PRO/CFS is colour-coded to make the results more comprehensible for patients and healthcare professionals in order to facilitate more active use of the PROMS during consultations. The active use of the PRO/CFS methodology that focuses on the feedback process is an important aspect of the FIT, as described by Kluger and DeNisi, ${ }^{18}$ as feedback has been found to improve communication between patients and healthcare professionals ${ }^{3}$ and to improve treatment outcomes. ${ }^{33} 34$ Patients have also emphasised the importance of feedback and discussion about their responses. ${ }^{7}$ Through this conversation the healthcare professional can address the topics and highlight whether the patient's responses have changed surprisingly in one way or another.

\section{Technology as a barrier}

As described by Bradley et $a l^{35}$ technology can be a barrier for patients and healthcare professionals. This might affect how patients respond to the questionnaires, as well as whether they complete the PRO/CFS prior to the consultation. However, our initial experiences are that the system is easy to $\log$ into and navigate. Most patients are familiar with using tablets in their daily life. As an attempt to overcome this potential barrier, we have secured technical support for the healthcare professionals, as well as training for them about using the system and interpreting the PRO/CFS results. A recent qualitative study which explored the implementation of Norse Feedback found that training and support were important for the clinicians to incorporate the PRO/CFS in consultation. 
The psychologists reported that learning and incorporating a new technological system was challenging in their everyday work, and characterised it as an overwhelming workload. ${ }^{36}$

\section{Strengths and limitations}

The PRO/CFS package chosen for this project needs to be adapted and validated for the population of patients in surgical treatment for obesity, as its relevance to the patients and healthcare professionals is crucial for the $\mathrm{PRO} / \mathrm{CFS}$ to be useful. ${ }^{37} \mathrm{~A}$ strength of this project is the involvement of both patients and healthcare professionals who, in collaboration with researchers, will design and evaluate the PRO/CFS. This involvement of patient representatives and outpatient nurses is considered important for producing the final version of the PRO/ CFS-thereby increasing the ecological validity of this project. Other strengths include tutoring healthcare professionals about how to incorporate the PRO/CFS in clinical conversations with patients, and the availability of both technical and methodological support.

The two outpatient clinics have organised their bariatric care differently, as the clinic at St. Olav Hospital has a mandatory course for the patients over 4 months before surgery. Furthermore, at Førde Hospital Trust the patients always have consultations with a nurse, and the surgeon meets the patients after 3 months and is consulted when it is beneficial for the patient. These differences in the organisation of bariatric care may strengthen the generalisability of the quantitative findings, as the results of the study will not be solely determined by the characteristics of a single clinic. Such differences may also contribute to richer findings in the qualitative inquiries. However, a potential limitation is that patients in one clinic meet primarily with the same healthcare professional, whereas patients at the other clinic meet with a larger number of healthcare professionals. It is possible that as more healthcare professionals are involved, there may be greater variability in how the PRO/CFS is used. We need to be aware of these differences when reporting results from the study. Furthermore, the recruitment of patients for the qualitative interviews may result in a selection bias. We will be aware of this possible bias when conducting the interviews and synthesising the findings, and report this as a potential limitation of the study.

In this project, we do not conduct a quantitative measure of how the healthcare professionals manage the feedback process, display provider empathy or demonstrate selfefficacy. This may represent a limitation. However, these aspects will be addressed during the qualitative inquiries.

\section{Future perspectives}

If we find that $\mathrm{PRO} / \mathrm{CFS}$ is appropriate in bariatric surgery follow-up, and that patients and healthcare professionals believe it adds value to the consultations, the effectiveness of the PRO/CFS will be tested on a larger scale, preferably as a randomised controlled trial. Whether some clinicians use feedback in a more effective way than others, demonstrate greater provider empathy, create a stronger working alliance or demonstrate self-efficacy will be important measures when we test the $\mathrm{PRO} / \mathrm{CFS}$ on a larger scale.

In conclusion, PRO/CFS may be a useful tool for the structured assessment of HRQOL and mental health before and after bariatric surgery. The consultations at the outpatient clinic are intended to be more patientcentred and may thus improve the follow-up rates over time at clinical consultations. As obesity is considered a complex chronic disease, and the positive effects of bariatric surgery go beyond weight loss in itself, a patientcentred model for follow-up is recommended. ${ }^{39}$ Ultimately, using PRO/CFS in clinical consultations may lead to improvements in patients' mental health and HRQOL after bariatric surgery.

\section{Author affiliations}

${ }^{1}$ Faculty of Health and Social Sciences, Western Norway University of Applied Sciences-Forde Campus, Forde, Norway

${ }^{2}$ Department of Global Public Health and Primary Care, University of Bergen Faculty of Medicine and Dentistry, Bergen, Norway

${ }^{3}$ Department of Family Medicine and Community Health, Duke University School of Medicine, Durham, North Carolina, USA

${ }^{4}$ Quality of Life Consulting, PLCC, Durham, North Carolina, United States

${ }^{5}$ Department of Psychiatry, Førde Hospital Trust, Forde, Norway

${ }^{6}$ Centre of Health Research, Førde Hospital Trust, Forde, Norway

Contributors PAH has been involved in planning, writing and editing of the entire manuscript. AA, RLK, CM, GST and JRA have contributed through editing the manuscript. JRA has also been involved in planning the study.

Funding The authors have not declared a specific grant for this research from any funding agency in the public, commercial or not-for-profit sectors.

Competing interests CM owns intellectual property in Norse Feedback, one of the measures used in the protocol.

Patient consent for publication Not required.

Provenance and peer review Not commissioned; externally peer reviewed.

Open access This is an open access article distributed in accordance with the Creative Commons Attribution Non Commercial (CC BY-NC 4.0) license, which permits others to distribute, remix, adapt, build upon this work non-commercially, and license their derivative works on different terms, provided the original work is properly cited, appropriate credit is given, any changes made indicated, and the use is non-commercial. See: http://creativecommons.org/licenses/by-nc/4.0/.

ORCID iD

Pål André Hegland http://orcid.org/0000-0001-6524-5744

\section{REFERENCES}

1 Boswell JF, Kraus DR, Miller SD, et al. Implementing routine outcome monitoring in clinical practice: benefits, challenges, and solutions. Psychother Res 2015;25:6-19.

2 Carlier IVE, Meuldijk D, Van Vliet IM, et al. Routine outcome monitoring and feedback on physical or mental health status: evidence and theory. J Eval Clin Pract 2012;18:104-10.

3 Hegland PA, Aasprang A, Hjelle Øygard S, et al. A review of systematic reviews on the effects of patient-reported outcome monitoring with clinical feedback systems on health-related quality of life-implications for a novel technology in obesity treatment. Clin Obes 2018;8:452-64.

4 Boyce MB, Browne JP, Greenhalgh J. The experiences of professionals with using information from patient-reported outcome measures to improve the quality of healthcare: a systematic review of qualitative research. BMJ Qual Saf 2014;23:508-18.

5 Philpot LM, Barnes SA, Brown RM, et al. Barriers and benefits to the use of patient-reported outcome measures in routine clinical care: a qualitative study. Am J Med Qual 2018;33:359-64. 
6 Sales CM, Alves PC. Patient-centered assessment in psychotherapy: a review of individualized tools. Clin Psychol 2016;23:265-83.

7 Börjesson S, Boström PK. "I want to know what it is used for": Clients' perspectives on completing a routine outcome measure (ROM) while undergoing psychotherapy. Psychother Res 2020;30:337-47.

8 Kendrick T, El-Gohary M, Stuart B, et al. Routine use of patient reported outcome measures (PROMs) for improving treatment of common mental health disorders in adults. Cochrane Database Syst Rev 2016;7:CD011119.

9 Lambert MJ, Whipple JL, Kleinstäuber M. Collecting and delivering progress feedback: a meta-analysis of routine outcome monitoring. Psychotherapy 2018;55:520-37.

10 de Jong K, van Sluis P, Nugter MA, et al. Understanding the differential impact of outcome monitoring: therapist variables that moderate feedback effects in a randomized clinical trial. Psychother Res 2012;22:464-74.

11 Kolotkin RL, Andersen JR. A systematic review of reviews: exploring the relationship between obesity, weight loss and health-related quality of life. Clin Obes 2017;7:273-89.

12 Jumbe S, Meyrick J. Contrasting views of the Post-bariatric surgery experience between patients and their practitioners: a qualitative study. Obes Surg 2018;28:2447-56.

13 Ghaferi AA, Lindsay-Westphal C. Bariatric surgery--more than just an operation. JAMA Surg 2016;151:232-3.

14 Busetto L, Dicker D, Azran C, et al. Practical recommendations of the obesity management Task force of the European association for the study of obesity for the Post-Bariatric surgery medical management. Obes Facts 2017;10:597-632.

15 Polit DF, Beck CT. Nursing Research : generating and assessing evidence for nursing practice. 10th ed. Philadelphia: Wolters Kluwer, 2017.

16 Staniszewska S, Brett J, Simera I, et al. GRIPP2 reporting checklists: tools to improve reporting of patient and public involvement in research. BMJ 2017;358:j3453.

17 O'Connell S, Palmer R, Withers K, et al. Requirements for the collection of electronic PROMS either "in clinic" or "at home" as part of the PROMs, PREMs and Effectiveness Programme (PPEP) in Wales: a feasibility study using a generic PROM tool. Pilot Feasibility Stud 2018;4:90.

18 Kluger AN, DeNisi A. The effects of feedback interventions on performance: a historical review, a meta-analysis, and a preliminary feedback intervention theory. American Psychological Association, 1996.

19 Aasprang A, Andersen JR, Våge V, et al. Psychosocial functioning before and after surgical treatment for morbid obesity: reliability and validation of the Norwegian version of obesity-related problem scale. PeerJ 2015;3:e1275.

20 Aasprang $\mathrm{A}$, Våge V, Flølo TN, et al. Patient-reported quality of life with obesity - development of a new measurement scale. Tidsskr Nor Laegeforen 201910.4045/tidsskr.18.0493. [Epub ahead of print: 20 Aug 2019].

21 Loge JH, Kaasa S, Hjermstad MJ, et al. Translation and performance of the Norwegian SF-36 health survey in patients with rheumatoid arthritis. I. data quality, scaling assumptions, reliability, and construct validity. J Clin Epidemiol 1998;51:1069-76.
22 Moltu C, Veseth M, Stefansen J, et al. This is what I need a clinical feedback system to do for me: a qualitative inquiry into therapists' and patients' perspectives. Psychother Res 2018;28:250-63.

23 Dimenäs E, Glise H, Hallerbäck B, et al. Well-Being and gastrointestinal symptoms among patients referred to endoscopy owing to suspected duodenal ulcer. Scand J Gastroenterol 1995;30:1046-52.

24 Flølo TN, Andersen JR, Nielsen HJ, et al. Translation, adaptation, validation and performance of the American weight efficacy lifestyle questionnaire short form (WEL-SF) to a Norwegian version: a crosssectional study. PeerJ 2014;2:e565.

25 de Jong K. Deriving implementation strategies for outcome monitoring feedback from theory, research and practice. Adm Policy Ment Health 2016;43:292-6.

26 Edbrooke-Childs J, Wolpert M, Deighton J. Using patient reported outcome measures to improve service effectiveness (UPROMISE): training clinicians to use outcome measures in child mental health. Adm Policy Ment Health 2016;43:302-8.

27 Braithwaite J, Churruca K, Long JC, et al. When complexity science meets implementation science: a theoretical and empirical analysis of systems change. BMC Med 2018;16:63.

28 Sjetne IS, Bjertnaes OA, Olsen RV, et al. The generic short patient experiences questionnaire (GS-PEQ): identification of core items from a survey in Norway. BMC Health Serv Res 2011;11:88.

29 Sullivan GM, Feinn R. Using effect Size-or why the $P$ value is not enough. J Grad Med Educ 2012;4:279-82.

30 IBM Corp. IBM SPSS statistics for windows, version 24.0. Armonk, NY: IBM Corp, 2016.

31 Malterud K. Systematic text condensation: a strategy for qualitative analysis. Scand J Public Health 2012;40:795-805.

32 Hill CE, Knox S, Thompson BJ, et al. Consensual qualitative research: an update. J Couns Psychol 2005;52:196-205.

33 Jensen-Doss A, Haimes EMB, Smith AM, et al. Monitoring treatment progress and providing feedback is viewed favorably but rarely used in practice. Adm Policy Ment Health 2018;45:48-61.

34 Delgadillo J, de Jong K, Lucock M, et al. Feedback-informed treatment versus usual psychological treatment for depression and anxiety: a multisite, open-label, cluster randomised controlled trial. Lancet Psychiatry 2018;5:564-72.

35 Bradley LE, Thomas JG, Hood MM, et al. Remote assessments and behavioral interventions in post-bariatric surgery patients. Surg Obes Relat Dis 2018:14:1632-44.

36 Hovland RT, Moltu C. Making way for a clinical feedback system in the narrow space between sessions: navigating competing demands in complex healthcare settings. Int $J$ Ment Health Syst 2019;13:68.

37 de Jong K. Challenges in the implementation of measurement feedback systems. Adm Policy Ment Health 2016;43:467-70.

38 Giangregorio L, Thabane L. Pilot studies and feasibility studies for complex interventions. An introduction. In: Richards DA, Hallberg IR, eds. Complex interventions in health : an overview of research methods. New York: Routledge, 2015: 127-35.

39 Fastenau J, Kolotkin RL, Fujioka K, et al. A call to action to inform patient-centred approaches to obesity management: development of a disease-illness model. Clin Obes 2019;9:e12309. 